Short communication

\title{
Cerebrospinal fluid and plasma lopinavir concentrations and viral response in virologically suppressed patients switching to lopinavir/ritonavir monotherapy once daily
}

Juan M Tiraboschi ${ }^{1 *}$, Hernando Knobel ${ }^{2}$, Arkaitz Imaz ${ }^{1}$, Judith Villar², Elena Ferrer ${ }^{1}$, Maria Saumoy ${ }^{1}$, Alicia González ${ }^{2}$, Nerea Rozas ${ }^{1}$, Antonia Vila ${ }^{1}$, Jordi Niubó ${ }^{3}$, Jordi Curto $^{1}$, Daniel Podzamczer ${ }^{1}$

${ }^{1}$ HIV Unit, Infectious Disease Department, Bellvitge University Hospital, L'Hospitalet, Barcelona, Spain

${ }^{2}$ Hospital del Mar, Barcelona, Spain

${ }^{3}$ Microbiology Service, Bellvitge University Hospital, L'Hospitalet, Barcelona, Spain

*Corresponding author e-mail: jmtiraboschi@bellvitgehospital.cat

\begin{abstract}
Background: Lopinavir/ritonavir (LPV/r) monotherapy is used in selected virologically-suppressed HIV-infected patients. Some would prefer a once-daily (OD) dose instead of the usual twice-daily dose to favour adherence. However, trough concentrations of the drug in blood and particularly in cerebrospinal fluid (CSF) may not be adequate to maintain viral suppression.

Methods: Prospective, open-label pilot study to evaluate the efficacy and safety of LPV/r monotherapy OD. HIV-1-infected patients, virologically suppressed for at least 6 months were enrolled. HIV viral load (VL) was determined at baseline and at weeks 4, 8, 12, 16, 24, 36, and 48. Lumbar puncture was performed in a subgroup of patients to evaluate CSF VL and CSF LPV concentrations.
\end{abstract}

Results: Twenty-one patients were included. At week 48, 85.7\% $(n=18)$ showed viral suppression ( $\mathrm{VL}<40$ copies $/ \mathrm{mL}$ ). Two patients had viral failure $(9.5 \%)$ and a third was withdrawn from the study because of gastrointestinal symptoms. Nine patients were enrolled in the substudy. CSF VL was $<40$ copies $/ \mathrm{mL}$ in all cases. Median (range) LPV concentration was $9.78 \mathrm{ng} / \mathrm{mL}$ (1.93-78.3) in CSF and 1970 (154$16700) \mathrm{ng} / \mathrm{mL}$ in plasma; the CSF/plasma ratio was 0.004 (0.001-0.186).

Conclusions: In this small pilot study, LPV/r monotherapy OD maintained plasma HIV RNA suppression at 48 weeks in most patients, with no cases of CSF viral escape. However, CSF LPV concentrations were close to the IC50 threshold in several patients; hence, this intervention should be avoided in patients with advanced immune suppression and/or those individuals presenting with significant co-morbidities such as Hepatitis $\mathrm{C}$ coinfection.

\section{Introduction}

A combination of three antiretroviral drugs is the standard of care for individuals with HIV infection. However it has been suggested that once HIV suppression is achieved, a single or dual active drug maintenance regimen might be effective in some patients[1-3]. Based on reasons such as avoiding potential NRTI toxicities, treatment simplification, and particularly, cost reduction, protease inhibitor monotherapy is considered a plausible option in several countries and is a recognised simplification option for selected patients in some guidelines (EACS, GESIDA)[4,5]. 
In contrast to the OD regimen of DRV/r, LPV/r monotherapy has the disadvantage of twice-daily administration, potentially limiting its use. Once-daily antiretroviral regimes may favour better adherence and virological suppression[6].

Although currently available data suffice to support the use of protease inhibitor monotherapy in selected patients, there is concern about the antiviral activity of this regimen at long term and in viral sanctuaries, such as the central nervous system (CNS)[7-9]. It is well recognized that plasma trough concentrations of LPV/r 800/200 $\mathrm{mg}$ OD are lower than the levels achieved with 400/100 mg BD [mean(SD), 3.62 (3.38) $\mathrm{ug} / \mathrm{mL}$ versus $7.13(2.93) \mathrm{ug} / \mathrm{mL}$ ], respectively[10]. While the OD dosage of $\mathrm{LPV} / \mathrm{r}$ associated with two nucleosides suffices to achieve plasma viral suppression in patients with wild type HIV-1[11], data are lacking regarding viral suppression with OD LPV/r monotherapy, and more importantly whether LPV/r monotherapy OD reaches acceptable CSF drug concentrations as when given BD (median $17 \mathrm{ng} / \mathrm{ml}$ IQR 12.1-22.7)[12] as well as its antiviral efficacy in the CNS.

The objective of this study was to prospectively evaluate the efficacy of LPV/r OD monotherapy in a group of virologically suppressed HIV-infected patients.

Cerebrospinal fluid (CSF) HIV-1 RNA levels and CSF LPV concentrations were investigated in a subgroup of these patients.

\section{Methods}

This is a prospective, open-label pilot simplification study to evaluate the efficacy and safety of LPV/r monotherapy OD, referred to as the KMON Study (NCT01581853). The patients enrolled were HIV-1-infected adults with plasma viral load (VL) $<40$ copies/mL for at least 6 months while on stable LPV/r monotherapy BD for more than 6 months. HIV VL was determined at baseline and at weeks 4, 8, 12, 16, 24, 36, and 48 thereafter. Treatment failure was defined as HIV VL $>40$ copies $/ \mathrm{mL}$ at any time point over follow-up, confirmed within the next 14 days, or discontinuation due to any reason.

In a subgroup of patients receiving LPV/r monotherapy OD for at least 1 month, a lumbar puncture was performed to assess CSF HIV RNA levels and CSF LPV concentrations.

Lumbar puncture was planned at 22 to 28 hours after the last LPV/r dose to determine the lowest concentration before the next dose. Two patients who were unable to have lumbar puncture in this time frame underwent the procedure at 11 hours after the last LPV dose. As in previously published studies [12;] LPV concentrations were compared with the LPV IC50 range derived from the control virus for the ViroLogic PhenoSense assay [median, $3.0 \mathrm{nmol} / \mathrm{l}(1.9 \mathrm{mg} / \mathrm{l})$ and 99 th percentile $8.1 \mathrm{nmol} / \mathrm{l}]$

HIV-1 RNA in plasma and CSF was determined by real-time polymerase chain reaction (m2000 Real Time System®, Abbot Molecular Inc., Des Plaines, IL; limit of detection, 40 copies $/ \mathrm{mL}$ ). Liquid chromatography-tandem mass spectrometry (Tandem labs, NJ) was used to determine CSF and plasma LPV concentrations. The Simplified Medication Adherence Questionnaire (SMAQ)[14] was completed at each visit.

Demographic and clinical characteristics were analyzed according to the nature of the variables: The results of continuous variables were described using the median and range and the results of categorical variables were described using frequencies and percentages. For comparisons between groups according to presence of virological failure and since variables did not display a normality distribution in continuous variables, Mann Whitney $U$ test was used, and in the case of qualitative 
variables, Fisher's exact test was used. For all statistical analyses, p-values less than or equal to 0.05 were considered significant. Analyses were performed using SPSS, version 19.0 (SPSS, an IBM company, Chicago, Illinois, USA).

\section{Results}

Twenty-one patients were included; $66.7 \%$ were men, median (range) age was 45.5 (35.4-57.4) years, and baseline and nadir CD4 counts were 648 (78-1285) and 154 (33-537) cells $/ \mathrm{mL}$, respectively. Almost half the patients (42.9\%) were HCV-positive. Patients had received LPV/r monotherapy BD for a median of 1.7 years $(0.7-7.5)$ before the study and had an undetectable viral load for a median of 3.5 years (0.59.5).

At week 48 , viral suppression ( $\mathrm{VL}<40$ copies $/ \mathrm{mL})$ was maintained in $85.7 \%(n=18)$ of patients. Three patients (14.3\%) had blips over follow-up: 2 patients had 2 separate episodes of transitory viral load rebound (76 and 74 copies $/ \mathrm{mL}$, and 381 and 800 copies $/ \mathrm{mL}$, respectively) and 1 patient had a single episode of viral rebound (713 copies $/ \mathrm{mL}$ ). None of these patients had viral failure after 48 weeks. Seven patients had a history of a viral load blip before starting on OD monotherapy, but only one of them showed evidence of a viral load blip during the study.

Two patients experienced viral failure (9.5\%). In both cases, antiretroviral treatment was switched to TDF/FTC/DRV/r OD and TDF/FTC/LPV/r OD, and both patients reached undetectable VL after the switch. A third patient was withdrawn from the study because of gastrointestinal symptoms (diarrhoea). There were no statistically significant associations of viral failure with nadir CD4 count, HCV coinfection, duration of undetectable viral load, or previous blips.

Nine patients were included in the CSF substudy. Lumbar puncture was performed a median (range) of 24 (8-36) weeks from baseline and 24 (11-28) hours after the last dose of LPV/r monotherapy OD. CSF HIV viral load was $<40$ copies $/ \mathrm{mL}$ in all patients, and was simultaneously undetectable in plasma. Median (range) LPV CSF concentration was 9.78 (1.9-78.3) ng/mL, plasma concentration was 1970 (154$16,700) \mathrm{ng} / \mathrm{mL}$, and the CSF/plasma ratio was $0.004(0.001-0.186)$ (Table 1).

\section{Discussion}

Most patients (85.7\%) switching from LPV/r monotherapy BD to OD administration in this study had an undetectable VL after 48 weeks of follow-up. These data are consistent with the findings of previous monotherapy studies[14], in which RNA suppression rates tended to be lower when switch equals failure criteria were used $(76 \%)$, and significantly higher when re-intensification was not considered failure (85\%)[1]. Two patients exhibited viral failure as per definition and a third was withdrawn from the study at 1 month of follow-up because of persistent diarrhoea. In all 3 patients, viral load was re-suppressed when nucleoside analogues were reintroduced or treatment was switched. Unfortunately, no resistance tests were available before re-suppression.

All patients but one reported excellent tolerance while receiving the once-daily regimen. Only one patient reported adherence $<95 \%$ during the study. Interestingly, this patient presented two non-consecutive blips (381 and 800 copies $/ \mathrm{mL}$ ), but had undetectable VL at week 48. A second patient also had two non-consecutive blips (74 and 76 copies $/ \mathrm{mL}$ ) and a third patient had a single blip (713 copies $/ \mathrm{mL}$ ). There were no concerns about treatment adherence in these last 2 patients.

Unpublished results from a small study $(n=10)$ using LPV/r monotherapy OD showed a high percentage of viral failures (4 of 9)[16]. One of the patients developed 
resistance-associated mutations, and for that reason, the study was interrupted prematurely. A second study (MONOCO 2012; $n=20$ ), looked at the efficacy and safety of LPV/r monotherapy once daily in a group of HIV-HCV co-infected patients who also received treatment adherence counselling[17]. Viral failure (defined as 2 consecutive HIV RNA determinations of $>50$ copies $/ \mathrm{mL}$ ) occurred in 7 patients. Viral suppression was documented in all patients after reintroduction of NRTIs. No resistance mutations were identified.

These two studies differ in several aspects from our trial. In both studies, all patients were receiving triple therapy before starting monotherapy OD, whereas in our study, all patients had been receiving monotherapy BD for a median of 1.7 years (range 0.7-7.5). Furthermore, all patients in the MONOCO study were coinfected with HCV. The authors concluded that most of the breakthrough cases could be explained by poor antiretroviral adherence. However, other studies have shown that HCV coinfected patients have a significantly lower virological response, suggesting that hepatitis $\mathrm{C}$ co-infection, likely associated with intravenous drug use, may be a marker of poor adherence[18]. In our cohort, almost half (42\%) the patients were HCV-positive; interestingly, the two patients who presented viral failure were HCV co-infected, although they were not active injection drug users.

As would be expected, LPV plasma concentrations were significantly lower with OD administration then with $\mathrm{BD}[11]$, but the values were still several times above the IC50. There were no cases of CSF viral escape in the cross-sectional sub-study, and LPV concentrations were above the IC50 for wild-type HIV-1 (mean IC50: $1.9 \mathrm{ng} / \mathrm{mL}$ in the absence of human serum). Median CSF LPV OD concentration was almost half of that when LPV/r is given BD. Four patients had LPV CSF values very close to the therapeutic threshold (within 5 times the IC50), which has been associated with viral escape with other drugs [19,20] (Figure 1).

All patients in our cohort had shown persistent viral suppression on BD monotherapy for several years before the study. At the end of the study, the results showed a high percentage of virologically suppressed patients $(85.7 \%)$. This could mean that these patients were, in general, good candidates not only for OD monotherapy, but also for protease inhibitor monotherapy, and that our design helped to predict a good virologic outcome in most patients.

The study limitations were a small sample size, the absence of a control arm and also the fact that neurocognitive assessments or inflammatory biomarkers in CSF were not carried out. Nonetheless, relevant data on CSF drug concentrations and viral replication were obtained, thus contributing to our knowledge regarding the use of antiretroviral therapy in HIV-infected patients.

In conclusion, this small pilot study performed in virologically suppressed patients receiving a $B D$ regimen of $L P V / r$ monotherapy indicates that switching to an $O D$ $\mathrm{LPV} / \mathrm{r}$ regimen can maintain plasma and CSF suppression at 48 weeks in a high percentage of these patients. Nonetheless, LPV CSF concentrations were close to the IC50 threshold in several cases, and this is a cause for concern. From our perspective this intervention should be avoided in patients with advanced immune suppression and/or those patients presenting with significant co-morbidities such as Hepatitis C co-infection.

\section{Acknowledgements}

This study was partially funded by the RD12/0017/0013 project, a part of the Plan Nacional $223 \mathrm{R}+\mathrm{D}+\mathrm{I}$, and was cofinanced by the ISCIII, Subdirección General de Evaluación y el 224 Fondo Europeo de Desarrollo Regional (FEDER). 
The preliminary results of this study were presented at the HIV Drug Therapy Conference, 2014 Glasgow, UK.

\section{Disclosures}

DP has received research grants or honoraria for advisories or conferences from Boehringer Ingelheim, GSK, ViiV, Pfizer, BMS, Abbott, Gilead, Janssen, Roche and Merck.

JT, HN, AI, JV, EF, MS, and AG have received consultancy fees or lecture sponsorships from Abbvie, BMS, Gilead Sciences, and ViiV Healthcare.

$\mathrm{NR}, \mathrm{AV}, \mathrm{JN}$ and JC: none to declare.

\section{References}

1. Arribas J, Girard PM, Paton N, et al. Efficacy of PI monotherapy versus triple therapy for 1964 patients in 10 randomised trials. J Int AIDS Soc. 2014;17:19788.

2. J.M. Gatell, J.R. Arribas, P.M. Girard, et al. Non-inferiority of dual-therapy (DT) with lopinavir/ritonavir (LPV/r) plus lamivudine (3TC) vs. triple-therapy (TT) with LPV/r plus two nucleos(t)ides (NRTIs) for maintenance of HIV viral suppression: 48-week results of OLE study. Abstract LBPE17, $20^{\text {th }}$ International AIDS Conference. Melbourne, Australia. 2014.

3. Burgos J, Crespo M, Falcó V, et al. Simplification to dual antiretroviral therapy including a ritonavir-boosted protease inhibitor in treatment-experienced HIV1-infected patients. J Antimicrob Chemother. 2012; 67:2479-86.

4. European Guidelines for treatment of HIV-infected adults in Europe. 2014 http://www.eacsociety.org/files/guidelines_english_71_141204.pdf

5. Enferm Infecc Microbiol Clin. 2014 Aug-Sep;32(7):447-58. doi: 10.1016/j.eimc.2014.02.018. Epub 2014 Jun 28. Executive summary of the GeSIDA/National AIDS Plan consensus document on antiretroviral therapy in adults infected by the human immunodeficiency virus (updated January 2014).

6. Parienti JJ, Bangsberg DR, Verdon R, et al. Better adherence with once-daily antiretroviral regimens: a meta-analysis. Clin Infect Dis. 2009;48:484-8.

7. Santos JR, Muñoz-Moreno JA, Moltó J, et al. Virological Efficacy in Cerebrospinal Fluid and Neurocognitive Status in Patients with Long-Term Monotherapy Based on Lopinavir/Ritonavir: An Exploratory Study. PLoS One. 2013; 8: e70201.

8. Gutmann C, Cusini A, Gunthard HF, et al. Randomized controlled study demonstrating failure of LPV/r monotherapy in HIV: the role of compartment and CD4-nadir. AIDS 2010 24: 2347-54.

9. Short communication: focal encephalitis related to viral escape and resistance emergence in cerebrospinal fluid in a patient on lopinavir/ritonavir monotherapy with plasma HIV-1 RNA suppression. Imaz A, Cayuela N, Niubó J, Tiraboschi JM, Izquierdo C, Cabellos C, Podzamczer D. AIDS Res Hum Retroviruses. 2014; 30:984-7.

10. Eron JJ, Feinberg J, Kessler HA et al. Once-daily versus twice-daily lopinavir/ritonavir in antiretroviral-naive HIV-positive patients: a 48-week randomized clinical trial. J Infect Dis. 2004 ;189:265-72.

11. 1.González-García J, Cohen D, Johnson M et al. Short communication: Comparable safety and efficacy with once-daily versus twice-daily dosing of lopinavir/ritonavir tablets with emtricitabine + tenofovir DF in antiretroviral- 
naïve, HIV type 1-infected subjects: 96 week final results of the randomized trial M05-730. AIDS Res Hum Retroviruses. 2010 ;26:841-5.

12. Capparelli EV, Holland D, Okamoto $C$ et al. Lopinavir concentrations in cerebrospinal fluid exceed the $50 \%$ inhibitory concentration for HIV. AIDS 2005; 19:949-52

13. Solas C, Lafeuillade A, Halfon P, Chadapaud S, Hittinger G, Lacarelle B. Discrepancies between protease inhibitor concentrations and viral load in reservoirs and sanctuary sites in human immunodeficiency virus-infected patients. Antimicrob Agents Chemother 2003; 47:238-243.

14. Knobel H1, Alonso J, Casado JL, et al. Validation of a simplified medication adherence questionnaire in a large cohort of HIV-infected patients: the GEEMA Study AIDS. $2002 ; 16: 605-13$.

15. Lopez-Cortes LF, Ruiz-Valderas R, Sánchez-Rivas E et al. Lopinavir Plasma Concentrations and Virological Outcome with Lopinavir-Ritonavir Monotherapy in HIV-1-Infected Patients., Antimicrob Agents Chemother. 2013; 57: 3746-3751.

16. Katner H; Kumar R; Nylund C et al. Lopinavir/Ritonavir Once Daily Monotherapy Pilot Study. The MONDAKAL Study. Poster \#1353. IDWeek 2012. San Diego, CA, USA.

17. Cooper $\mathrm{C}$, la Porte $\mathrm{C}$, Tossonian $\mathrm{H}$ et al. A pilot, prospective, open-label simplification study to evaluate the safety, efficacy, and pharmacokinetics of once-daily lopinavir-ritonavir monotherapy in HIV-HCV coinfected patients: the, MONOCO study. HIV Clin Trials. 2012;13:179-88.

18. Arribas JR, Montes ML, Prakash M, et al: The MONET trial: correlation between hepatitis $\mathrm{C}$ co-infection and HIV RNA responses during darunavir/ritonavir monotherapy, for patients with HIV RNA $<50$ copies/mL at baseline. ISHEID 2010. Abstract O-15.

19. Calcagno A, Yilmaz A, Cusatoc J et al. Determinants of darunavir cerebrospinal fluid concentrations: impact of once-daily dosing and pharmacogenetics. AIDS. 20; 26: 1529-153

20. Di Yacovo MS, Moltó J, Ferrer E , et al. Antiviral activity and CSF concentrations of $600 / 100 \mathrm{mg}$ of darunavir/ritonavir once daily in HIV-1 patients with plasma viral suppression. J Antimicrob Chemother. 2015. [Epub ahead of print] 